\title{
A High-Frequency Electronically Tunable Quadrature Oscillator
}

\author{
C. J. M. Verhoeven
}

\begin{abstract}
A new architecture of mutually coupled regenerative oscillators is presented. It has been used to design a lownoise high-frequency VCO capable of producing two output signals in quadrature with essentially identical properties. The phase relation between the quadrature outputs is frequency independent and extremely stable. A new way of coupling the regenerative oscillators is suggested in order to improve the frequency stability of the coupled oscillator system. Results obtained from a test chip have verified the viability of the new oscillator concept. The oscillator circuit has been realized in a medium-frequency $\left(f_{T}=3 \mathrm{GHz}\right)$ bipolar process. The tuning range extends to $500 \mathrm{MHz}$. At an oscillation frequency of 200 $\mathrm{MHz}$, measured phase noise was $-121 \mathrm{dBc} / \mathrm{Hz}$ at $1-\mathrm{MHz}$ distance from the carrier.
\end{abstract}

\section{INTRODUCTION}

$\mathrm{T}$ HERE is a large demand for integratable high-frequency electronically tunable oscillators. Regenerative (or relaxation) oscillators are completely integratable. In a lot of telecommunication applications, signals that are in quadrature are needed (e.g., in synchronous detectors). Then the accuracy and stability of the phase relation between the two signals is of great importance. Much research has already been done on the topic of phase noise in regenerative oscillators, and several low-noise designs have been presented [1]-[4]. In this paper an oscillator topology is presented that produces two output signals in quadrature with identical properties (same level, same phase noise, etc.). The phase relation between the two signals is accurate, frequency independent, and extremely stable.

Very often quadrature signals are generated using divider circuits, while the oscillator driving the dividers has to operate on twice the desired output frequency. This inevitably increases the power consumption. Moreover, the maximum output frequency feasible in the available IC process will be reduced by a factor of 2 . An alternative approach is based on the use of a comparator to derive a quadrature signal from the zero crossings of the capacitor voltage. However, then the properties of the two output signals are not identical and the phase relation is neither exact nor very stable.

The disadvantages of the quadrature oscillators described above are not present in the oscillator concept presented in this paper. The quadrature signals can be generated at the maximum operating oscillation frequency,

Manuscript received December 11, 1991; revised February 26, 1992 The author is with the Department of Electrical Engineering, Delft University of Technology, $2628 \mathrm{CD}$ Delft, The Netherlands.

IEEE Log Number 9200275.

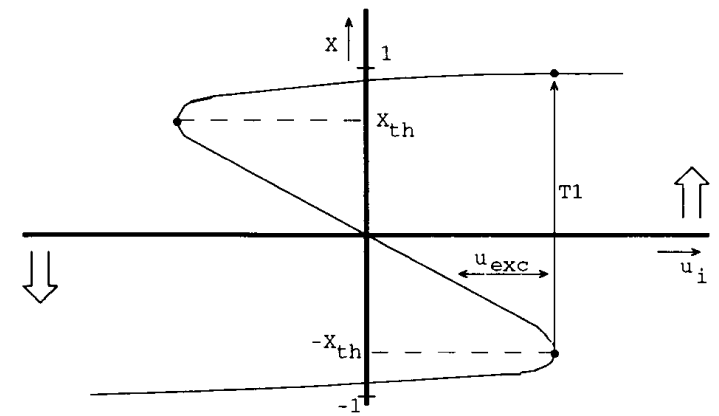

Fig. 1. Input signal of a Schmitt trigger versus its output signal $(X)$.

with a quality that is much better than that of the oscillator-comparator concept. This makes the new concept very useful for the generation of high-performance highfrequency quadrature signals. The oscillator presented consists of a pair of mutually coupled regenerative oscillators, but at the output terminals it can be regarded on as one oscillator. A $200-\mathrm{MHz}$ version, based on the wellknown emitter-coupled oscillator, has been realized in a medium-frequency bipolar IC process $\left(f_{T}=3 \mathrm{GHz}\right)$. It is merely intended to be a demonstrator of the viability of the concept. The essential new aspect of the concept is the way the coupling is established, resulting in an accurate quadrature relation (patented).

\section{The Basic Oscillator}

The basic regenerative oscillator used in this application consists of a capacitor, a controlled current source, and a Schmitt trigger [3]. Fig. 1 depicts a typical plot of the input signal $\left(u_{i}\right)$ of a Schmitt trigger versus its state $X$ (normalized output signal). In the case of a regenerative oscillator, $u_{i}$ is the capacitor voltage. The "steady-state curve" connects operating points of the Schmitt trigger in which it is in equilibrium, so it does not change state when it is in an operating point that is on the curve. It can be shown that when the operating point of the Schmitt trigger is not on the curve, it changes state [1]. The state changes in the positive direction when the operating point is at the right side of the curve and it changes in the negative direction when the operating point is on the left side of the curve. In the region where the loop gain of the Schmitt trigger is larger than unity $\left(-X_{t h}<X<X_{t h}\right)$, the slope of the steady-state curve is negative. The excitation $\left(u_{e x c}\right)$ is defined as the horizontal distance between the operating point and the curve [1]. Suppose the operating point is on the curve and the loop gain is larger than unity. When the 
input signal is changed, which causes the operating point to move away horizontally from the curve, the operating point starts moving away vertically from the curve. From Fig. 1 it can be seen that in the regenerative region, the Schmitt trigger tends to increase its excitation (self-excitation [1], [2]). The operating point keeps moving vertically until it enters a region where the loop gain is smaller than unity. There it will move towards the curve, so finally it ends up at some other part of the curve. This has been illustrated with trajectory $T 1$ in Fig. 1.

\section{The Coupled Oscillator Circuit}

When the capacitor voltage crosses a reference level, the Schmitt trigger switches the charging current. Eventually, this causes the capacitor voltage to drop below the threshold level again. Then the external cause for the Schmitt trigger to switch is removed and it depends on its excitation whether it will either continue or abort its transition. This "negative feedback mechanism" can deteriorate the noise performance of the oscillator considerably [1]. Unfortunately, the presence of the negative feedback loop is essential in the oscillator, since it causes the capacitor voltage to "bounce back" at the threshold levels, thus establishing the desired periodical signal. The oscillator should be designed such that the negative feedback loop has no effect on the timing of the transitions and thus on the noise susceptibility of the Schmitt trigger.

In the coupled oscillator system comparators are used to detect the crossing of some "virtual" threshold level by the capacitor voltage, a predefined time before the true threshold level is crossed. After a well-defined delay the crossing of this virtual threshold level causes a transition of the Schmitt trigger. This delay is derived from a second identical oscillator running on the same frequency but not in phase with the original oscillator. This second oscillator is also equipped with a comparator that measures virtual threshold levels, and the required time delay is defined by the first oscillator. In this way a system of two mutually coupled oscillators is created. The switching of a comparator has no immediate effect on its input signals, so the negative feedback loop is effectively broken. Fig. 2 shows the basic circuit diagram of the coupled oscillator. It consists of two regenerative oscillators and two comparators. The oscillators consist of a capacitor $\left(C_{A, B}\right)$ and a Schmitt trigger. The two oscillator-comparator pairs have identical parameters. The comparators switch at the zero crossings of the capacitor voltages. The output signal of comparator $\boldsymbol{A}$ is added to the capacitor voltage of oscillator $B$ and vice versa. In steady state the zero crossings of oscillator $A$ initiate the transitions in oscillator $B$ and vice versa.

In practice the two oscillators to be coupled usually do not have exactly equal periods, but in the coupled system, they are forced to be equal. Below this will be explained graphically. In Fig. 3 idealized steady-state curves of one of the oscillators have been depicted. The dashed lines indicate the threshold levels of the Schmitt trigger. When

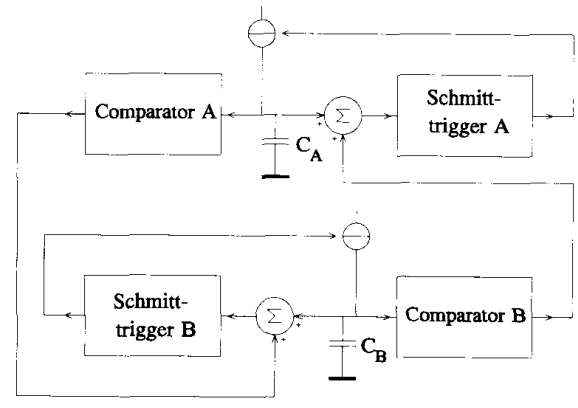

Fig. 2. The basic coupled oscillator.

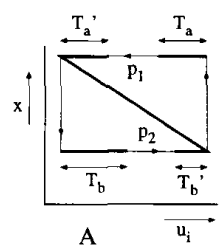

(a)

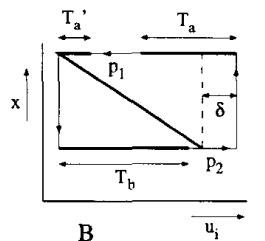

(b)

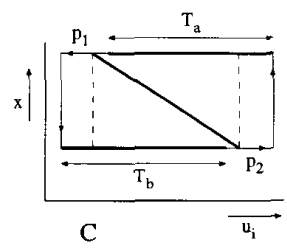

(c)
Fig. 3. The steady-state lines of an oscillator with added signals $p_{1}$ and $p_{2}$.

the input signal $u_{i}$ crosses these levels, the Schmitt trigger switches. The operating point moves counterclockwise in a rectangle at different speeds for different parts of the rectangle.

Horizontally the operating point normally moves with a speed that is determined by the changing rate of the capacitor voltage (trajectories $T_{a, b}, T_{a, b}^{\prime}$ ). It is determined by the capacitor value and the charging current. Only when a comparator switches, and thus a fast voltage change occurs at the input of the Schmitt trigger, does the operating point move with the switching speed of the comparator (trajectories $p_{1,2}$ ). Usually this is much faster than the changing rate of the capacitor voltage. The noise susceptibility of the Schmitt trigger is decreased very much when it is because of this fast signal that the input signal of the Schmitt trigger crosses a threshold level since the excitation of the Schmitt trigger is increased [1], [2].

At the vertical parts the operating point moves with the switching speed of the Schmitt trigger.

In first-order approximation the period is equal to the time the operating point spends moving horizontally along trajectories $T_{a, b}$ and $T_{a, b}^{\prime}$.

The trajectories $p_{1}$ and $p_{2}$ are introduced by the output signal of a comparator that is added to the capacitor voltage. Their size is determined by the amplitude $u_{p}$ of the comparator output signal. During $p_{1}$, the comparator switches to a state in which its output signal decreases the input signal of the Schmitt trigger, and during $p_{2}$, it switches to a state in which the input signal is increased. This can increase or decrease the period of the oscillator depending on the place where $p_{1}$ and $p_{2}$ occur in the diagram. 
Suppose the two oscillators are not in quadrature yet and the other oscillator has a larger period. In Fig. 3(a) the period of the oscillator is the shortest. The threshold levels are crossed due to the changes of the capacitor voltage itself. Then, each subsequent period, the $p_{1}$ and $p_{2}$ move further counterclockwise in the state diagram, because their timing is related to the period of the other (slower) oscillator. Finally, during $p_{2}$ a transition is initiated. This situation has been depicted in Fig. 3(b). Then an extra time $\Delta T=2 \delta\left(C / I_{\text {charge }}\right)$ is added to the period of the oscillator. Hence, the oscillator is slowed down. When the other oscillator is still slower, $p_{1}$ and $p_{2}$ keep on moving counterclockwise. Then eventually, also during $p_{1}$, a transition will be initiated, which causes a further increase of $\Delta T$ (Fig. 3(c)). When the periods of the two oscillators have become equal, the movement of $p_{1}$ and $p_{2}$ stops. Then the oscillators are in lock. When both oscillators are in a mode that corresponds to Fig. 3(c), so all transitions are initiated by the comparator signals, a lownoise behavior can be expected [1].

Until now, the effect of the synchronizing signals has been discussed for only one of the two oscillators. In the other oscillator, a similar effect is present with-because that oscillator is the slowest $-p_{1}$ and $p_{2}$ moving clockwise.

Each oscillator has a free-running period, $T_{\text {free }}$, which is the period of the oscillator when no injected signals are present. In the situation as depicted in Fig. 3(a), $T_{\text {free }}$ is decreased with a time $T_{d e c}$ when $p_{1}$ is at the top and $p_{2}$ is at the bottom, and it is increased with a time $T_{i n c}$ when $p_{2}$ is at the top and $p_{1}$ is at the bottom. With $u_{p}$ being the amplitude of the injected signal it follows that

$$
T_{i n c}=T_{d e c}=\frac{2 u_{p} C}{I_{\text {charge }}} .
$$

In order to make locking in quadrature possible, the difference between the free-running periods of the oscillators should not differ more than $T_{i n c}+T_{d e c}$.

When the free-running periods of the oscillators are equal, an exact quadrature coupling is obtained. Then the state diagrams of both oscillators are identical. When the free-running periods are not equal, a small phase error exists which depends on the switching speed of the injected signals. It is proportional to the timing differences between the injected signals in the two state diagrams of the oscillators. Since the matching of components in an integrated circuit is good and the injected signals are generally fast, the phase error can be very small $\left(<<1^{\circ}\right)$.

When the coupled oscillator system is modulated, measurements showed that lock is not lost or even measurably disturbed. Hence, the coupled oscillator system can be used as a $\mathrm{CCO}$ with output signals that have an accurate quadrature relation. This phase relation is independent of the operating frequency of the coupled oscillator. It merely depends on the symmetry between the two oscillators. Advantageous properties of normal regenerative oscillators, like linearity and a large tuning range, are also present in the coupled oscillator.

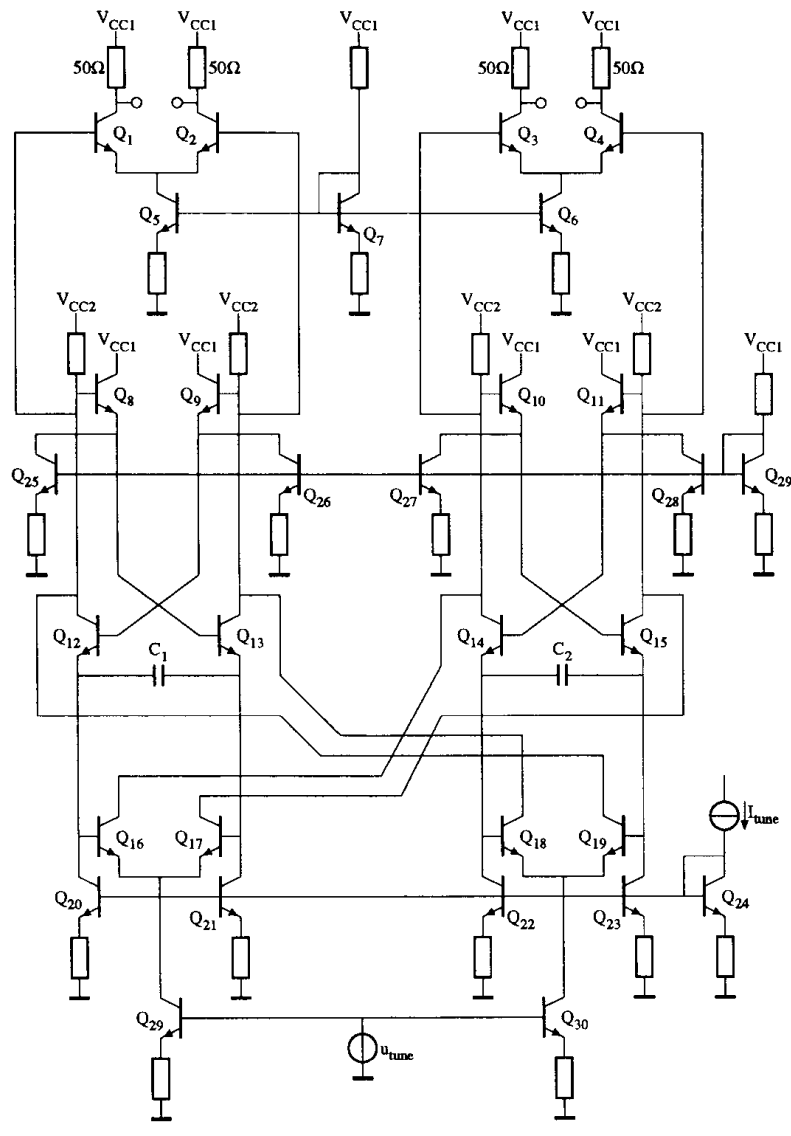

Fig. 4. Circuit diagram of the complete circuit.

\section{Semicustom Realization and Experimental RESULTS}

Fig. 4 shows a circuit diagram of the complete circuit. The basic regenerative oscillators are formed by $C_{1}, Q_{12,13}$ and $C_{2}, Q_{14,15}$, respectively, together with the resistors in the collector paths of these transistors. Two output buffers necessary for the measurement purposes have been implemented around $Q_{1,2}$ and $Q_{3,4}$. The two comparators have been implemented as differential pairs $Q_{16,17}$ and $Q_{18,19}$. The output currents of the comparators are injected into the corresponding oscillators where they are converted into a voltage by the resistors. This has the same effect as directly adding a voltage to the capacitor voltage. The other components are for biasing purposes.

The oscillator has been realized on a medium-frequency bipolar semicustom array and functions well from very low frequencies up to more than $500 \mathrm{MHz}$. The phase noise spectrum has been measured using special phase noise measurement equipment (HP3048A), at a $200-\mathrm{MHz}$ carrier frequency. The phase noise spectrum decreases $-20 \mathrm{~dB} /$ decade over a large frequency range, which is in accordance with the phase noise expressions derived in [1] and [3]. The absolute value of the phase noise is -121 $\mathrm{dBc} / \mathrm{Hz}$ at $1-\mathrm{MHz}$ distance from the carrier. The stability 
of the quadrature relation could not be measured due to the noise floor of the available phase detector, but this fact indicates that the stability is very good. The complete circuit, inclusive of the output buffers, consumes about 29 $\mathrm{mA}$ at a supply voltage of $8 \mathrm{~V}$. The actual oscillator core consumes about $14 \mathrm{~mA}$. The total timing capacitance for one oscillator is about $3 \mathrm{pF}$ at an output frequency of $200 \mathrm{MHz}$.

\section{CONCLUSION}

The design considerations for a new integratable coupled regenerative quadrature oscillator have been described. The feasibility of the oscillator concept has been shown by means of a semicustom realization. The quadrature relation has proven to be very accurate and stable.
The circuit functions well over a large frequency range up to $500 \mathrm{MHz}$. It was not attempted to make a low-noise or a low-power design in this feasibility study, and it is expected that a redesign will result in an even better noise performance at a lower power consumption.

\section{REFERENCES}

[1] C. J. M. Verhoeven, "First-order oscillators," thesis, Delft Univ. Technology, Delft, The Netherlands, 1990

[2] C. J. M. Verhoeven, "A new model for regenerative circuits," in Proc. Midwest Symp. Circuits Syst. (Syracuse, NY), 1987, pp. 631-634.

[3] J. G. Sneep and C. J. M. Verhoeven, "A new low-noise 100-MHz balanced relaxation oscillator," IEEE J. Solid-State Circuits, vol. 25, no. 3, pp. 692-698, 1990.

[4] A. A. Abidi and R. G. Meyer, "Noise in relaxation oscillators," IEEE J. Solid-State Circuits, vol. SC-18, no. 6, pp. 794-802, 1983 